\title{
An equation of average lifetime of the minority carriers in semiconductors from photo-electrochemical measurement
}

\author{
M. Muhibbullah ${ }^{\mathrm{a}}$, M. Golam Mowla Choudhury ${ }^{\mathrm{a}}$, Sharif M Mominuzzaman ${ }^{\mathrm{b}}$, Junie Jhon \\ $\mathrm{M}$. Vequizo ${ }^{\mathrm{c}}$ and Yang $\mathrm{Kai}^{\mathrm{c}}$ \\ ${ }^{a}$ Department of Electronics and Telecommunication Engineering, \\ Daffodil International University, 102 Shukrabad, Dhanmondi, Dhaka-1207, Bangladesh, \\ Tel-phone: +88 029116774 Ex-133 \\ e-mail: m.muhammad.205@nitech.jp (M. Muhibbullah), gmcw9f@daffodilvarsity.edu.bd (Golam Mowla). \\ ${ }^{\mathrm{b}}$ Department of Electrical and Electronic Engineering, \\ Bangladesh University of Engineering and Technology (BUET), Dhaka-1000, Bangladesh \\ Phone: +88-02-8611594; +8802-9665650 80/Ext. 7770 (Off), 7113 (Dept) Fax: +88-02-8611594, \\ e-mail: momin@eee.buet.ac.bd. \\ ${ }^{c}$ Department of Engineering Physics, Electronics and Mechanics, \\ Nagoya Institute of Technology, Gokiso, Showa, Nagoya 466-8555, Japan \\ Tel-phone and Fax: +81 0527355581 , \\ e-mail: cih16508@stn.nitech.ac.jp (J. J. M. Vequizo) dengen2jp@yahoo.co.jp (K. Yang).
}

In a semiconductor, the average lifetime of the minority carriers is important for the applications of optoelectronic devices. Longer and shorter average lifetimes are expected for different applications. Photo-electrochemical measurement shows electrical and optical properties with some information of average lifetime of the minority carriers. An equation of the average lifetime for excited minority carriers in the semiconductors is derived from the results of photo-electrochemical measurement.

Key words: average lifetime, minority carrier, PEC measurement

\section{GENERAL INTRODUCTION}

\subsection{Introduction}

Identifying the factors affecting the optical properties of the semiconducting materials is important for opto-electronic device applications. Photoelectrochemical (PEC) measurement is one of the simple methods available to identify the optical characteristics of the semiconductors. In this method, the materials semiconducting type and photosensitivity can be revealed and estimated [1-9]. In PEC measurements, some carriers are generated within the semiconductor during illumination condition. In the absence of the light energy the excited carriers recombine very quickly. Although the lifetime as well as average lifetime of an excited minority carrier is very short but average lifetime is different for different materials. Average lifetime of the minority carriers is an important property needed for different kind of semiconductor applications. For instance, longer average lifetime is necessary in photovoltaic cells and shorter average lifetime is needed for sensor applications.

\subsection{Photo-electrochemical measurement}

In photo-electrochemical (PEC) measurement, a semiconducting material and electrolyte solution are in series and biased with small applied potential in the semiconductor electrode. The photocurrent response is measured and plotted against voltage or time under light and dark conditions. The details of the PEC process are available in the doctoral theses $[10,11]$. Briefly, an incident light from a lamp source is focused and irradiated from the transparent side of a semiconductor material. The incident light is mechanically turned on and off every few seconds in an alternate manner using a shutter situated between the light source and the semiconductor. The PEC measurement could be performed under either by applying linearly increasing potential or constant biasing.

Under illumination, some carriers are excited in the illuminated region of the semiconductor. Then the excited minority carriers are diffused to the surface during their lifetime to participate in the electrochemical reaction at the electrolyte-semiconductor interface. When a certain light intensity irradiates on the semiconductor, the amount of the excited minority carriers is equal to the amount of the excited majority carriers, but the photocurrent density is different [1-9] during anodic and cathodic biases. The PEC measurement reveals that electrons and holes move under cathodic and anodic biasing, respectively. This is why larger current under anodic bias and no or very little current under cathodic bias for p-type semiconductor in a dark condition are obtained [1-3]. The electrons of cathode electrode cannot reach up to anode electrode through the electrolyte solution. Local ions of the electrodes keep the role for the current flow. The ions release or receive the electrons from the electrodes and become neutral. In a small bias voltage, some of the ions are consumed. The involved ions (anions and cations) increased with increasing voltage. For instance, if the carrier concentration in the semiconductor is less than the ion concentration $(\mathrm{Ni})$, then the origin of the observed current is dominantly from the semiconductor. This series path is like a gate. The carrier concentration in the semiconductor or ion concentration in the electrolyte controls the flow of the current. However, the priority is either the low amount of carrier concentration or low ion concentration. At room temperature, ion concentration is constant for a constant 
solution concentration then, the impedance of the electrolyte (Zso) is constant. Variation of total impedances (Zse + Zso) under bias condition depends on the type of biasing (either anodic or cathodic) and on the semiconductor impedance (Zse), that is, the donors and acceptors concentration of an extrinsic semiconductor. In dark condition, the value of impedance for n-type semiconductor (carrier only electron) under cathodic bias is less than the value of impedance of the same semiconductor when anodic bias is applied. In the dark condition, the n-type semiconductor exhibits large current during cathodic biasing and no (or very little) current under anodic bias (carrier only hole) because minority carriers are almost zero in this condition [4-8]. Under illumination condition and anodic bias, some photogenerated currents are exhibited in the n-type semiconductor. These photogenerated currents are larger than the dark current because minority carriers are increased by the light energy. Imagine that the photogenerated carrier concentration is $N$, if it is less than the ions concentration $N i$ (it is expected). Then, the nature of current density should be for semiconductor. But, in the cathodic bias very little or no photocurrent is increased under light condition because majority carrier concentration of the n-type semiconductor is larger than the ion concentration $N_{i}$ (it is expected) in light conditions also in dark. So, the output current density appears with nature of the current for the ions concentration. Hence, increasing the photo-generated carriers during cathodic bias does not necessarily increase the photocurrent.

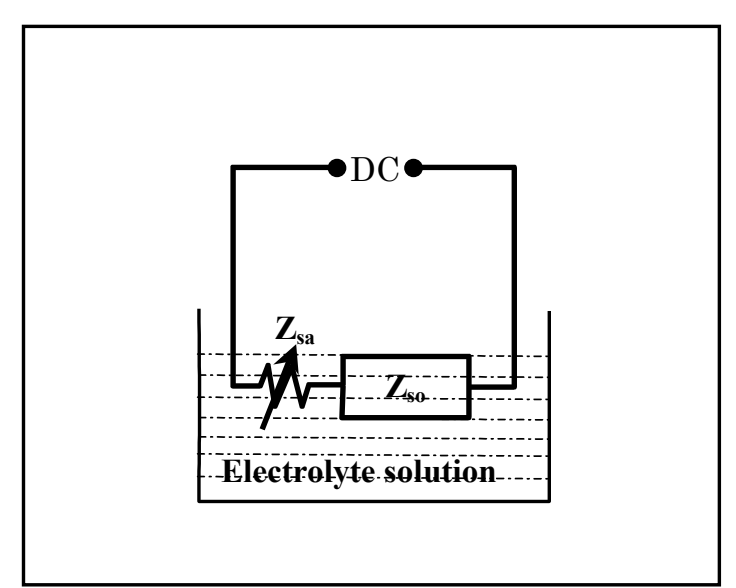

Fig. 1: The impedance of the PEC cell.

The number of carriers that participate in the electrochemical reaction in the semiconductor increases with applied potential for a constant light intensity. The number of participate carriers as well as the photocurrent become maximum for a certain value of voltage (minimum voltage $\mathrm{V}_{\min }$.). In this minimum voltage all the induced carriers are utilized to flow the current. To calculate the average lifetime of the induced minority carriers, the current density should be measured by applying this (minimum) constant voltage to the semiconductor similar to a PEC cell experiment $[12,13]$. Current is taken under dark condition for few seconds after irradiation. In the dark condition the induced carriers decrease exponentially until equilibrium.

Some semiconductors are very electrochemically active [5-7] in a certain electrolyte solution. In this case, the value of $V_{\min }$ is zero or near zero. Impedances also depend on the difference of energy levels between the carriers of semiconductor and valance band of the ions in the electrolyte solution. During transition from semiconductor to electrolyte, the carriers absorb or release the energy, then the impedance increase or decrease, respectively. It is observed that the current density of excited carriers under dark condition decreases exponentially for $\mathrm{n}$-type semiconductor.

Consider the following parameters: $N_{d}$ and $N_{a}$ as donor and acceptor concentrations repectively. $N_{e}$ as photogenerated electron and $N_{h}$ as photogenerated hole concentrations of a semiconductor. Then, the following relations can be obtained:

For p-type semiconductor:

$$
\frac{N_{e}}{N_{e}+N_{d}}>\frac{N_{h}}{N_{h}+N_{a}}
$$

since $N_{e}=N_{h}$ and $N_{d}<N_{a}$

For n-type semiconductor:

$$
\frac{N_{e}}{N_{e}+N_{d}}<\frac{N_{h}}{N_{h}+N_{a}}
$$

since $N_{e}=N_{h}$ and $N_{d}>N_{a}$

For intrinsic semiconductor:

$$
\begin{aligned}
& \frac{N_{e}}{N_{e}+N_{d}} \approx \frac{N_{h}}{N_{h}+N_{a}}, \\
& \text { since } N_{e}=N_{h} \text { and } N_{d} \approx N_{a}
\end{aligned}
$$

\subsection{Brief background of the average lifetime}

There is a popular equation [14] of lifetime with respect to capture cross-section, carrier thermal velocity and concentration of carrier recombination centers in the semiconductor. Experimental measurement of lifetime is very difficult using this equation. Another equation is used to measure the minority carrier lifetime for $\mathrm{p}-\mathrm{n}$ junction $[15,16]$ by measuring the storage time, constant forward current and constant reverse current. Ronald A. Sinton et al [17] have determined the average lifetime of minority carrier of a semiconductor using steady-state photo conductance technique. In their method the photoconductance, current density, electron and hole mobility are measured experimentally with presence of light.

In this work, we have derived the average lifetime equation of the minority carriers for a semiconductor with respect to the decay of current density (of dark), which can be observed in the results of PEC measurement and the derived form of average life time equation is different from the reported equations.

\section{THEORY}

In the irradiation condition, some carriers such as electrons and holes are generated in a semiconductor by absorption of the light energy. When light irradiates on a semiconductor, the carriers generation rate $G$ starts to increase and its maximum value is attained within a short time. The carrier recombination rate $R$ follows the generation rate with $G>R$ and quickly equilibrates $(G=$ $R$ ) and becomes constant after a certain time. Similarly, in the dark condition the carrier generation rate $G$ starts 
to decrease exponentially and it reaches a minimum value within a short time. The recombination rate $R$ follows the generation rate $G$ with $G<R$ and quickly it becomes equilibrium $(G=R)$ after a certain time for dark condition. The carriers collides each other randomly and in this way the recombination are being obstructed. The number of collision decreases with the reduction of carriers.

We have derived the equation of average lifetime of minority carriers for dark and non-equilibrium conditions. Experimental results of induced photo current density with voltage [1-9] and with time $[12,13]$ are available in previous works. In the non-equilibrium state (i.e. suddenly disappoint from the light) of dark condition the induced carriers $N$ decrease with time by the recombination. The current (current density) changes as carrier concentration does. For a semiconductor of the PEC measurement we can write,

Current $(\mathrm{I})\{=\mathrm{JA}\}=$ Voltage $(\mathrm{V}) \times \frac{1}{\operatorname{Impedance}\left(Z_{s a}\right)}$

Impedance of a semiconductor depends (inverse proportional) on its carrier concentration when bias voltage and other parameters (electrolyte solution, solution concentration, temperature etc.) are constant. In this situation the current (current density) is linearly proportional with the carrier concentration. So, the photocurrent density $J$ and induced carrier concentration $N$ in a semiconducting material can be written as;

$$
|J| \propto|N|
$$

Considering the absolute value,

or, $J=C N$

$C$ is the proportionality constant.

or, $\frac{I}{A}=C N$

$\mathrm{I}$ is the current and $\mathrm{A}$ is the irradiated area of the semiconductor.

$$
\begin{aligned}
\text { or, } \frac{\frac{\partial Q}{\partial t}}{A}=C N \\
\frac{e \partial N}{\partial t}=C N, e \text { is the electronic charge. }
\end{aligned}
$$

Here $\partial N$ is the small number of carriers that react with the electrolyte to flow current at small $\partial t$ time. Therefore,

$$
\frac{\partial N}{\partial t}=\frac{A C N}{e}
$$

A minus sign should be added in the above equation because the number of induced carrier concentration $N$ decreases with time under non-equilibrium dark condition.

Hence,

$$
\frac{\partial N}{\partial t}=-\left(\frac{A C}{e}\right) N
$$

or, $\frac{\partial N}{\partial t}=-K N$

here $K=\frac{A C}{e}$

From eqn. (6), we get,

$\frac{\partial N}{N} \equiv-K \partial t$

or, $\ln N=-K t+C$

when $t=0$ then $N=N_{o}$,

so, $C=\ln N_{o}$

and, $\ln \frac{N}{N_{o}}=-K t$

or, $N=N_{o} e^{-K t}$

Putting the value of $N$ from equation (5) to equation (9), we get

$$
J=J_{o} e^{-K t}
$$

This equation agrees with the experimental results $[12,13]$ of induced photocurrent density with time in non-equilibrium dark condition.

From equation (9), we get

$$
t=\frac{1}{K}\left(\ln N_{o}-\ln N\right)
$$

According to the equation (9) the carrier concentration $N$ with time $t$ (n-type semiconductor under constant anodic bias) is drawn in Fig. 2. The current density $J$ is proportional to the carrier concentration $N$ in the semiconductor. Hence, the current density decreases exponentially with time as expressed in equation 10 , similar to the carrier concentration $N$ as seen in Fig. 2. This means that the natures of the current density and carrier concentration with respect to time are similar.

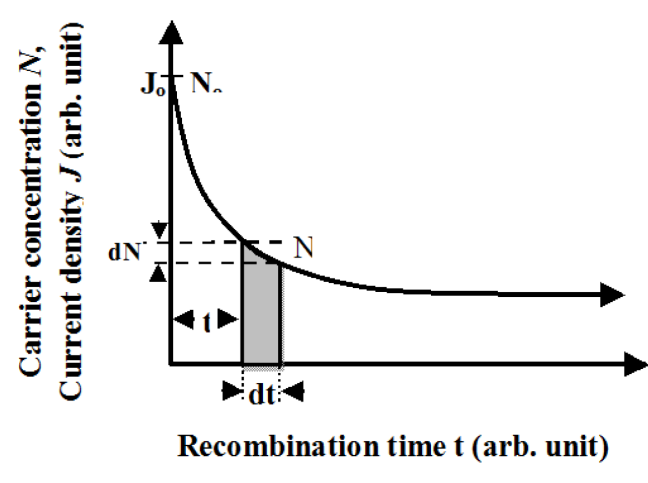

Fig. 2: Excited minority carrier concentration or current density with recombination time in non-equilibrium under dark condition.

In n-type semiconductor with anodic bias, the carriers are holes that are minority carriers as explained in introduction part. To derive the equation of average 
lifetime of minority carriers let us consider the amount of initial carriers (holes) concentration $N_{o}$ which become $N$ for the time duration of $t$ sec. (as depicted in Fig. 2). Then a small number $d N$ of the carriers recombine for the small time $d t$ sec. If $d t$ is very short i.e. $d t \rightarrow 0$ then we can say that the lifetime of the number of $d N$ carriers is equal to $t$ sec. The summation of the lifetimes of $d N$ carriers is $(d N \times t)$ sec. and the average lifetime of $d N$ carriers can be expressed as:

$$
d \tau=\frac{(d N \times t)}{d N} \text { (sec.) }
$$

So, the average lifetime of the minority carriers for considering all carriers is given by:

$$
\tau_{a v}=\frac{\int_{N_{o}}^{0} t d N}{\int_{N_{o}}^{0} d N}
$$

or, $\tau_{a v}=\frac{\int_{N_{o}}^{0} t d N}{-N_{o}}$

Using the equation, $t=\frac{1}{K}\left(\ln N_{o}-\ln N\right)$, we get,

$$
\tau_{a v}=\frac{1}{-K N_{o}}\left(\ln N_{o} \int_{N_{o}}^{0} d N-\int_{N_{0}}^{0} \ln N d N\right)
$$

or, $\tau_{a v}=\frac{1}{K}$

From equation (11) and (15), we get

or, $t=\tau_{a v}\left(\ln N_{o}-\ln N\right)$

From equation (5) and (16),

$t=\tau_{a v}\left(\ln \left(\frac{J_{o}}{C}\right)-\ln \left(\frac{J}{C}\right)\right)$

or, $t=\tau_{a v}\left(\ln J_{o}-\ln J\right)$

Hence, from equation (17), we can find the average lifetime of a semiconductor from the slope of the graph of $\left(\ln J_{o}-\ln J\right)$ against time. Also, the induced (photosensitivity) carrier concentration for different semiconducting materials can be compared by calculating $N$ and using the experimental values of $J$ and $A$ for given intensity of light.

\section{CONCLUSION}

An equation of the average lifetime for excited minority carriers in the semiconductors is derived from the results of PEC measurement. The average lifetime can be measured by the equation. The equation may help in the perfect application of a semiconductor in the opto-electronic devices.

\section{ACKNOWLEDGEMENT}

The authors are grateful to Dr. Ashraf M. Abdel Haleem of Fayoum University, Fayoum, Egypt for his useful discussions.

\section{REFERENCES}

[1] Muhammad Muhibbullah and Masaya Ichimura, Transactions of the Materials Research Society of Japan, 36[2], (2011), 195-198.

[2] Muhammad Muhibbullah and Masaya Ichimura, Japanese Journal of Applied Physics, 49 (2010), 081102-1 to 081102-4.

[3] M. Muhibbullah, M. Ichimura / Materials Research Bulletin 47 (2012) 1968-1972.

[4] A.M. Abdel Haleem, M. Ichimura, Thin Solid Films 516 (2008) 7783-7789.

[5] Ashraf M. Abdel Haleem and Masaya Ichimura, Japanese Journal of Applied Physics 48 (2009) 035506.

[6] A.M. Abdel Haleem, M. Ichimura, Materials Science and Engineering B 164 (2009) 180-185.

[7] M. Gunasekaran, P. Ramasamy and M. Ichimura, Journal of Electrochemical Society, 153 [7] (2006) G664-G668.

[8] Naglaa Fathy and Masaya Ichimura, Journal of crystal Growth 294 (2006), 191-196.

[9] Kaoru OMOTO, Naglaa FATHY and Masaya ICHIMURA, Jpn. J. Appl. Phys., 45[3A] (2006) 1500-1505.

[10] Muhammad Muhibbullah, Doctoral Thesis, Nagoya Institute of Technology, 2012.

[11] Ashraf M. Abdel Haleem Hassan, Doctoral Thesis, Nagoya Institute of Technology, 2010.

[12] Teketel Yohannes and O. Inganas, Solar Energy Materials and Solar Cells 51 (1998) 193-202.

[13] Yanzhong Hao, Maizhi Yang, Chen Yu, Shengmin Cai, Minsheng Liu, Louzhen Fan and Yongfang Li, Solar Energy Materials \& Solar Cells 56 (1998) 75-84.

[14] A. S. Grove, Physics and Technology of Semiconductor Devices, Printed by John Wiley and Sons, Inc., 1967.

[15] H. J. Kuno, IEEE Trans. Electron. Devices, 11 (1964) 8-14.

[16] Yeonwoong Jung, Aleksandar Vacic , Daniel E. Perea, Samuel T. Picraux , and Mark A. Reed, Adv. Mater. 23 (2011) 4306-4311.

[17] Ronald A. Sinton and Andres Cuevas, Appl. Phys. Lett., 69 [17] (1996) 2510-2512.

(Received December 20, 2012; Accepted May 24, 2013) 